\section{CONHECIMENTO DE TÉCNICOS DE ENFERMAGEM SOBRE O MÉTODO CANGURU NA UNIDADE NEONATAL}

\author{
Nursing technicians'knowledge of the Kangaroo-Mother Care \\ method in the neonatal unit
}
Conocimiento de técnicos de enfermería sobre el método Canguro en la unidad neonatal

Artigo Original

\section{RESUMO}

Objetivo: Identificar o conhecimento dos técnicos de enfermagem sobre a aplicação do Método Canguru (MC) e analisar sua importância na assistência na Unidade de Tratamento Intensivo Neonatal. Métodos: Pesquisa descritiva com abordagem qualitativa, realizada com 20 técnicos de enfermagem em uma unidade neonatal. Os dados foram coletados no período de março a abril de 2012 através da aplicação de entrevistas semiestruturadas e analisados através do método ídeo-central. Resultados: Evidenciou-se que os técnicos de enfermagem possuem conhecimento sobre o método canguru, entretanto, relatam dificuldades em identificar suas etapas no cotidiano da prática neonatal; acreditam ser importante na assistência, pois ajuda na recuperação, no crescimento e no desenvolvimento do recém-nascido; além de promover a participação dos pais, proporcionando uma assistência humanizada. Conclusão: Os técnicos de enfermagem investigados conhecem o MC e sua importância na assistência neonatal, embora apontem dificuldades quanto à identificação das suas etapas e a sua aplicabilidade em seus cotidianos.

Descritores: Método Canguru; Recém-nascido; Enfermagem Neonatal.

\section{ABSTRACT}

Objective: To identify the nursing technicians'knowledge about the Kangaroo-Mother Care Method (KMC) implementation and analize its importance for assistance in the Neonatal Intensive Care Unit. Methods: Descriptive research with qualitative approach, held with 20 nursing technicians in a neonatal unit. Data was collected in the period from March to April 2012 by carrying out semi-structured interviews and analized through the central-idea method. Results: It was evidenced that the nursing technicians have knowledge about the kangaroo method; however, they report difficulties in identifying its steps in their everyday neonatal practice; they believe it is important in health care, as it helps in the newborn's recovery, growth and development; in addition to promoting the parents' participation, providing humanized care. Conclusion: The investigated nursing technicians know the KMC and its importance in neonatal assistance, although they point out difficulties regarding the identification of its steps and applicability in their daily practice.

Descriptors: Kangaroo-Mother Care Method; Infant, Newborn; Neonatal nursing.

\section{RESUMEN}

Objetivo: Identificar el conocimiento de técnicos de enfermería sobre la aplicación del Método Canguro (MC) y analizar su importancia en la asistencia de la Unidad de Tratamiento Intensivo Neonatal. Métodos: Investigación descriptiva de abordaje cualitativo realizada con 20 técnicos de enfermería en una unidad neonatal. Los datos fueron recogidos entre marzo y abril de 2012 con la aplicación de entrevistas semi-estructuradas y analizados con el método ideo-central. Resultados: Se evidenció que los técnicos de enfermería tienen conocimiento sobre el método canguro, sin embargo, relatan dificultad para identificar
1) Universidade Estadual do Pará - UEPA Belém (PA) - Brasil
Recebido em: 01/03/2013

Revisado em: 21/07/2013 Aceito em: 21/09/2013 
sus etapas del cotidiano de la práctica neonatal; creen en su importancia en la asistencia ya que ayuda en la recuperación, el crecimiento y el desarrollo del recién-nacido; además de promover la participación de los padres, promoviendo una asistencia humanizada. Conclusión: Los técnicos de enfermería investigados conocen el MC y su importancia en la asistencia neonatal, aunque apunten dificultades sobre la identificación de sus etapas y su aplicabilidad en el cotidiano.

Descriptores: Método Madre-Canguro; Recién-Nacido; Enfermeria Neonatal.

\section{INTRODUÇÃO}

O parto prematuro, devido o seu crescente aumento a cada ano em muitos países subdesenvolvidos, tem-se caracterizado como um problema de saúde pública. Avanços tecnológicos e humanísticos vêm sendo apresentados para o aumento da expectativa de sobrevida dos recém-nascidos, destacando-se neste cenário o Método Canguru (MC) como uma estratégia de atenção perinatal ${ }^{(1)}$.

Com base nas pesquisas já realizadas e nos relatos de experiência de diferentes serviços, estudiosos concluíram que o MC tem potencial para melhorar a saúde e a sobrevivência dos recém-nascidos com baixo peso, particularmente onde os recursos são limitados, devido ao seu basto custo ${ }^{(2)}$.

No Brasil, a partir do final da década de 90, esta preocupação traduziu-se na "Atenção Humanizada ao Recém-Nascido de Baixo Peso - Método Canguru" (AHRNBP-MC). Elaborada e implementada pelo Ministério da Saúde, em 12 de julho de 2007, pela portaria 1.683 e através de normas, protocolos e de um amplo processo de capacitação nas diferentes regiões do país, caracterizando-se principalmente pela mudança na forma do cuidado neonatal ${ }^{(3)}$, sendo o primeiro país a padronizar seus procedimentos através de estratégias que promovessem cursos de treinamento padrão para as equipes de profissionais de enfermagem ${ }^{(4)}$.

O MC, popularmente conhecido como "cuidado mãe canguru" ou "contato pele a pele", semelhante aos marsupiais ${ }^{(2)}$, é uma alternativa ao cuidado neonatal convencional para bebês de baixo peso ao nascer, devido possibilitar um melhor prognóstico ao recém nascido (RN), estimulando o cuidado humanizado, que busca fortalecer o laço entre mãe e bebê, incentivando o aleitamento materno e a competência materna ${ }^{(5,6)}$.

Sua metodologia divide-se em três etapas distintas, iniciando pela identificação do trabalho de parto prematuro, com o RN apresentando peso inferior a 1500 gramas, o que impede uma apresentação conjunta à mãe, precisando, dessa forma, ser internado na unidade de tratamento intensivo neonatal (UTIN). Durante esse período, a mãe e a família devem ser orientadas em relação às condições da criança ${ }^{(7)}$.

Deve-se explicar a importância do método e do acesso precoce e livre dos pais à UTIN, sendo o contato pele-apele estimulado logo que as condições clínicas do bebê permitirem. Nesta primeira etapa deve-se voltar para a prestação de todas as informações e ensinamentos à mãe, ao pai e à família. Isso acontece antes de se iniciar o método. Nessa fase, a família é orientada quanto à saúde do e à importância da família e do $\mathrm{MC}^{(8)}$.

Durante a segunda etapa, o RN, que se encontra na UTIN, geralmente apresenta uma situação clínica estável. Nessa situação, já pode ficar, continuamente, acompanhado de sua mãe, devendo o RN encontrar-se com estabilidade clínica, com um peso mínimo de $1.250 \mathrm{~g}$ e estar em alimentação materna plena, sonda gástrica ou copo, o que possibilita o ganho de peso mínimo diário maior que $15 \mathrm{~g}$. Já na terceira e última etapa, a criança recebe alta hospitalar, sendo seu acompanhamento realizado periodicamente através de consultas ambulatoriais até que o bebê atinja o peso de $2.500 \mathrm{~g}^{(9)}$.

Para garantir os resultados positivos do método, o acompanhamento ambulatorial deve garantir a assistência ao bebê por médicos e outros membros da equipe multidisciplinar como: enfermeiros, fisioterapeutas, psicólogos, nutricionistas, fonoaudiólogos e assistentes sociais. Por isso, é de suma importância que o método seja realizado da maneira mais correta possível. Através de orientações adequadas dos profissionais da equipe que prestam assistência nos acompanhamentos hospitalares, domiciliar e ambulatorial ${ }^{(10)}$.

O profissional técnico de enfermagem, neste cenário, atua integralmente na primeira etapa do método, implementando a estimulação do RN e o fortalecimento do vínculo com a família, através de um cuidado individualizado que promova a diminuição dos estressores ambientais, além fornecer orientações fundamentais para a mãe neste momento inicial, como a produção de leite materno, equilíbrio emocional, confiança e controle das reações do $\mathrm{RN}$, removendo o medo e a insegurança na habilidade de cuidar do novo $\operatorname{ser}^{(10,11)}$.

De acordo com a Lei $\mathrm{N}^{\circ} 7498$ de 25 de junho de 1986, o técnico de enfermagem exerce atividade de nível médio, envolvendo orientação e acompanhamento do trabalho de enfermagem em grau auxiliar e participação do planejamento da assistência de enfermagem, cabendo-lhe, especialmente, participar da programação da assistência de enfermagem e executar ações assistenciais de enfermagem, como as preconizadas pelo AHRNBP-MC(4). 
Apesar do esforço do Ministério da Saúde na capacitação desses profissionais, nas maternidades e na normatização do programa canguru, a sua implantação nos serviços mostrou-se complexa. A experiência inicial de implantação analisada pelo Ministério da Saúde salientou que após o período de capacitação, de grande empolgação da equipe, o funcionamento efetivo do programa apresentava problemas. Um: ficava limitado pelas práticas hospitalares globais e, assim, discordantes das preconizadas pelo método. Dois: revelava o pouco entendimento dos gestores e das equipes de suporte ${ }^{(10,12)}$.

$O$ enfermeiro enfrenta algumas barreiras na implementação do método. Uma delas é a resistência da parte dos profissionais em aceitar inteiramente a proposta como um projeto de cunho interdisciplinar, que tenha real impacto na qualidade global da assistência neonatal ${ }^{(11)}$. Outro ponto é a insensibilidade desses para colocar em prática o MC. Nesta perspectiva inclui-se desde questões relacionadas à sensibilização para realizar o acolhimento às mães/famílias dos $\mathrm{RN}$ prematuros e/ou com baixo-peso que entram na unidade e optam pelo desenvolvimento do canguru, até a exposição de dificuldades em envolver os pais no dia a dia da unidade ${ }^{(13)}$.

Com base no exposto, foi levantado o questionamento: "Qual o conhecimento que o profissional técnico de enfermagem tem sobre o Método Canguru?" Esta investigação justifica-se no fato de que os preceitos jurídicos afirmados através da política do MC capacitam o profissional para se tornar habilitado na humanização dos cuidados neonatais, no acolhimento das famílias e na estimulação do vínculo afetivo. No entanto, será que estes profissionais estão realmente cientes do desenvolvimento destas atribuições? Frente a estes questionamentos, o presente estudo tem por objetivo identificar o conhecimento dos técnicos de enfermagem sobre a aplicação do Método Canguru e analisar sua importância na assistência na Unidade de Tratamento Intensivo Neonatal = UTIN .

\section{MÉTODOS}

Trata-se de um estudo descritivo com abordagem qualitativa $^{(14)}$, realizado na Unidade de Terapia Intensiva Neonatal (UTIN) da Fundação Pública Estadual Hospital de Clinicas Gaspar Viana, em Belém do Pará, Brasil, referência no atendimento ao $\mathrm{RN}$ que necessita de assistência especializada e de alta complexidade.

Atualmente, a presente unidade é composta por 30 técnicos de enfermagem; destes, 20 aceitaram participar da pesquisa após serem apresentados aos objetivos. Fizeram parte do estudo os participantes que tinham no mínimo três anos de atuação na referida UTIN e que participaram da capacitação para atuar no MC. Obedecendo aos preceitos éticos, foram utilizados nomes codificados para os entrevistados, garantindo anonimato aos sujeitos da pesquisa. Os nomes foram dispostos de acordo com a ordem crescente de participação e representados pela letra "T", a saber: T1, T2, T3 e assim sucessivamente.

A coleta dos dados ocorreu entre março a abril de 2012, através de entrevista semiestruturada, orientada por um roteiro contendo: a) perguntas sobre as variáveis sociais como: sexo, idade, tempo de atuação na unidade e grau de escolaridade; b) perguntas acerca de seus conhecimentos sobre o método, a aplicabilidade e a sua importância para a assistência. A coleta era interrompida quando nem um dado novo estava sendo acrescentado ao estudo, ou seja, quando os dados começaram a ser repetidos, obedecendo assim ao princípio da saturação ${ }^{(15)}$.

As informações foram agrupadas e em seguida analisadas através da modalidade ídeo-central, análise temática que visa evidenciar ideias centrais ou ideiaschaves nucleares dos discursos; ideias que emergem das respostas obtidas questão por questão. Ao final da análise, consegue-se reunir as ideias-chaves, resignificando-as, reagrupando-as e/ou reorganizando-as em outros núcleos de sentido formando quadros ${ }^{(16)}$, as quais são apresentadas e discutidas a seguir, dentro das categorias que emergiram dos dados: "Descortinando o conhecimento do técnico de enfermagem sobre o método canguru" e "O técnico de enfermagem frente à implementação do método canguru".

Ressalta-se que o presente estudo respeitou os aspectos éticos presentes na Resolução n¹96/96, do Conselho Nacional de Saúde, com assinatura do Termo de Consentimento Livre e Esclarecido e obteve aprovação do Comitê de Ética em Pesquisa da Fundação Pública Estadual Hospital de Clinicas Gaspar Viana, sob protocolo $n^{\circ} 002 / 2012$.

\section{RESULTADOS E DISCUSSÃO}

Em sua totalidade, os sujeitos eram do sexo feminino, com idade mínima de 25 anos e máxima de 44 anos, com tempo de atuação na unidade entre quatro a quinze anos, com grau de escolaridade variado, sendo: quatro com nível superior completo, sete com nível superior incompleto e nove com nível médio.

As ideias centrais das participantes apresentaram um universo até então desconhecido. Estudos abordam o conhecimento do MC sob a perspectiva do enfermeiro ${ }^{(3-6,13)}$, entretanto, ainda não foi explorada a perspectiva dos profissionais técnicos de Enfermagem sobre a temática; esta perspectiva será apresentada e discutida a seguir. 


\section{Descortinando o conhecimento do técnico de enfermagem sobre o método canguru}

As depoentes, ao serem indagadas sobre o que significava o MC em sua assistência, 12 (60\%) revelaram que o $\mathrm{MC}$ está relacionado com a promoção do vínculo afetivo, como observado a seguir:

"[...] ajuda o bebê prematuro a ficar mais próximo da mãe, não só da mãe, mas do pai, dos avós ou algum parente que tenha uma ligação mais próxima do bebê $[\ldots]$ '...(T9)

“[...] Pode ser aplicado por qualquer membro da família, tanto pai como a mãe, e colocado perto da mãe ou do pai, pele a pele, ele fica aconchegado [...]”.'(T13)

Os relatos apresentam-se condizentes com as informações encontradas em outros estudos ${ }^{(5,17)}$ nacionais que avaliaram a aplicação do $\mathrm{MC}$ e seus benefícios para os RNs. Os participantes destes estudos, assim como os entrevistados na presente pesquisa, assimilaram o método como uma estratégia que proporciona o estabelecimento do vínculo afetivo entre os pais e o bebê, o fortalecimento do vínculo mãe-filho e a promoção da confiança que é adquirida pelos pais no cuidado de seu bebê $\hat{e}^{(13)}$.

A descoberta das necessidades da mãe e do pai, assim como as do próprio bebê, de usufruir da presença do filho durante a hospitalização, tem sido um dos ganhos do MC, o que, no presente estudo diferiu dos demais estudos dispersos na literatura nacional ${ }^{(17,18)}$.

Os depoentes da presente pesquisa relataram que a presença do pai traz, para todos, vivências de uma estrutura familiar positiva para a recuperação do bebê, sendo esta indispensável, mesmo em situação de crise, podendo ser mantida pelas funções relacionais de cuidado e afetividade. Se para o bebê internado esses parceiros trazem a narrativa da história familiar, para os irmãos e os avós sua participação reforça o valor dos vínculos familiares que, neste momento, são reconhecidos pela equipe do hospital como capazes e necessários na medida de intervenção no $\mathrm{RN}^{(10)}$.

O estabelecimento e a manutenção do vínculo durante o período de hospitalização é fundamental para o despertar do cuidado da família para com o bebê, como também, para acelerar o processo de recuperação de sua saúde. Fato que consolida e equipara o conhecimento dispersado nas respostas dadas pelas entrevistadas da presente pesquisa com outro estudo ${ }^{(17)}$.

Quanto à humanização do cuidado ao RN, 6 (30\%) das depoentes do atual estudo salientaram que o MC é indispensável à oferta de um cuidado humanizado, como evidenciado nos discursos abaixo:

\footnotetext{
“[...] É uma forma de humanização em questão ao manuseio, uma forma de fazer os cuidados, dos
}

procedimentos, que tem que ter toda uma técnica, todo um processo, visando o $R N$, o que ele está sentindo e passando nesse momento [...]”. (T8)

"É um método que faz acomodação das crianças, fazendo com que elas se sintam aconchegadas apesar de ser um ambiente altamente estressante, fazendo com que tenham o máximo de conforto, manter a criança no ninho alinhada [...]".(T15)

A visão de cuidado anunciada por estes profissionais desvenda a forma como dispensam seus cuidados, mais intrinsecamente, a forma de assistir o RN no MC. Evidenciase a humanização como uma modalidade de cuidado diretamente relacionada ao MC, sendo uma estratégia que contempla a tendência da humanização e da integralidade do cuidado, que sinaliza que a assistência não deve ser somente dirigida ao bebê, mas, também, a toda a sua família, pois serão eles que assumirão o cuidado do RN no domicílio ${ }^{(3)}$. O $\mathrm{MC}$, como o conjunto de assistência humanizada oferecida, desde o nascimento, ao RN, aos pais e aos outros membros da família, promove o primeiro encontro entre o bebê e a família e dá, aos pais, a oportunidade de escolher o tempo em que permanecerão com o bebê na posição canguru. Esse período deve ser prazeroso e capaz de possibilitar intimidade à dupla: mãe-bebê ou pai-bebê $\hat{e}^{(19)}$.

Para o Ministério da Saúde (MS), humanizar o atendimento ao RN significa, entre outros, ter segurança técnica de atuação profissional, ou seja, eficácia na atenção ao RN, condições hospitalares compatíveis com o período neonatal e para a participação da família no processo assistencial. Tudo isso, aliado à necessidade da atenção individualizada ${ }^{(10)}$. Os relatos das entrevistadas na presente pesquisa mostram atitudes coerentes e que vão de encontro às ações preconizadas pelo MS. Porém, é importante ressaltar que os profissionais atuantes no ambiente neonatal devem se atentar às necessidades de aprender e entender a "linguagem do prematuro", pois, assim, é possível avaliar diariamente a necessidade individual destes, melhorando a conduta assistencial ao RN e sua família.

A amamentação é um dos benefícios do método canguru, importante para o binômio mãe-filho, tanto do ponto de vista físico quanto do psicológico; neste caso, o aleitamento é entendido como um ato de amor, que exige carinho, atenção, paciência e disponibilidade da mãe para com o RN. É um compromisso da mãe com seu filho, que proporciona carinho e saúde, tornando o vínculo mãe e filho mais intenso ${ }^{(2)}$.

O MC estimula a amamentação do $\mathrm{RN}$, pois se entende que o leite humano possui características nutritivas e bioquímicas necessárias para conferir ao leite uma composição ideal. Dessa forma, contribui para o crescimento e desenvolvimento do RN, sendo seguro, pois oferece ao RN proteção contra infecções e garante parte da imunização, 
auxiliando no desenvolvimento de estímulos sensoriais auditivos, táteis e emocionais sem anormalidades ${ }^{(2,12)}$.

Assim, as evidências teóricas ${ }^{(2,13,19)}$ exploradas nesta categoria, sob a ótica dos profissionais de enfermagem, convergem para o entendimento de que, quanto mais cedo o RN for colocado na mama, maior será a chance de se estabelecer o vínculo mãe-filho e menor é a possibilidade do desmame precoce. Este fato ressalta a importância da aplicação do MC para a recuperação eficiente do RN e a prestação de cuidados cada vez mais individualizados.

Quanto às etapas do método, 8 (40\%) das entrevistadas na atual pesquisa apresentaram dificuldades em relatar e identificar a operacionalização das etapas, como exemplificado abaixo:

"[...] O método possui três etapas, não sei dizer detalhadamente essas etapas." (T1)

"[...] sei que são três etapas, mas não sei dizer explicadinho todas."(T6)

"[...] são três etapas que a gente tenta desenvolver a primeira etapa, mas não sei as etapas detalhadamente". (T16)

Este fato chama a atenção para o agravo da assistência dispensada, pois todas as investigadas realizaram previamente o curso de capacitação - Atenção Humanizada ao Recém-nascido de baixo peso - o que leva a perceber que a capacitação foi importante para o início do processo de implantação do $\mathrm{MC}$, entretanto, pode não ter sido suficiente para a implantação das três fases do método, como recomendado pelo MS.

A implementação das diretrizes preconizadas pelo MS pressupõe equipes de saúde com habilidades não só para orientar a prática do $\mathrm{MC}$ nas unidades neonatais, mas também, para lidar com os aspectos que podem influenciar $\mathrm{o}$ ato de cuidar no âmbito da família ${ }^{(18)}$. Dessa forma, para um desenvolvimento adequado do método canguru, o profissional de enfermagem necessita conhecer, assumir e por em prática o seu papel neste cuidado, o que não foi evidenciado no presente estudo, pois a prática do método se convergiu somente na primeira etapa.

Outros mecanismos que certamente seriam necessários para atingir este objetivo, estão vinculados ao ato de sustentabilidade das práticas, promoção de supervisão, financiamento, fornecimento de material educativo, troca de experiências e seleção adequada dos profissionais para a capacitação ${ }^{(20)}$.

\section{O Técnico de enfermagem frente à implementação do método canguru}

Nesta categoria, apresentam-se as relações realizadas pelas depoentes quanto à aplicabilidade do método na
UTIN, e $16(80 \%)$ afirmam que aplicam o método dentro de varias estratégias do seu dia-dia, como: diminuição do barulho, da luz ambiente, posicionamento do $\mathrm{RN}$ e incentivo à adoção da posição canguru, conforme demonstrado nos depoimentos a seguir:

“[...] gente proporciona um ambiente calmo e tranquilo porque nós sabemos que o barulho próximo ao $R N$, principalmente o de baixo peso, atrapalha na recuperação $[\ldots] "(T 1)$

"[...] o soninho é respeitado para não ficar manuseando, a gente procura colocar o paninho, a gente procura mexer com ele em conjunto, enfermeiro e técnico, a gente faz isso porque os estudos comprovam que quando desorganizam, eles perdem peso [...]" (T11).

"[...] Coloco "rolinho" pra ele se sentir bem aconchegado e aquecido [...]" (T13).

O ambiente da UTIN proporciona uma experiência ao $\mathrm{RN}$ bastante diferente daquela vivenciada no período intrauterino, pois este ambiente possuía características distintas do útero materno como: temperatura agradável e constante, o aconchego e os sons extrauterinos que outrora eram filtrados consideravelmente ${ }^{(16)}$.

Diminuir barulhos e luz ambiente é fundamental para uma boa recuperação do RN. Assim como ressaltado pelas entrevistadas, estudos ${ }^{(10,12,17)}$ mostraram que os menores pacientes se desenvolvem melhor quando o barulho é minimizado, o volume do monitor é diminuído ou trocado por outro tipo de alarme, e quando a luz direta é reduzida, os bebês prematuros têm menor risco para o desenvolvimento da retinopatia da prematuridade. Além disso, os níveis constantes de luz podem tornar o desenvolvimento normal do ciclo sono-vigília mais lento ${ }^{(19)}$. Nesse sentido, um ambiente extrauterino inadequado, caracterizado por situações constantes de estresse, manipulação, privação do sono, ruído e luminosidade excessiva, resulta em efeitos aversivos ao desenvolvimento adequado do sistema nervoso e é indicador de risco para o desenvolvimento normal do $\mathrm{RN}^{(2)}$.

A perspectiva das participantes sobre a manutenção de um bom ambiente na UTI e sua contribuição para o RN é condizente com os descritos pelo MS, o qual afirma que reduzir os estímulos do ambiente é uma medida importante para o melhor desenvolvimento do paciente ${ }^{(10)}$. Fato este também evidenciado em outro estudo que referenciou que $73 \%$ das participantes acreditam na influência do ambiente de forma mínima ou moderada na dor e na recuperação ${ }^{(21)}$.

$\mathrm{O}$ acolhimento é o ato de receber e atender os diferentes integrantes da família do bebê internado na unidade neonatal, procurando facilitar sua inserção nesse ambiente. $\mathrm{O}$ acolhimento envolve uma ação não somente física, mas também de cunho afetivo ${ }^{(12)}$. Sabe-se que, para o nascimento 
antes do termo, esse contato inicial pode não ocorrer logo após o nascimento. Portanto, cabe à equipe de saúde tentar proporcionar um contato inicial em um ambiente acolhedor, com o objetivo de proporcionar um contato íntimo dos pais com seu bebê(10). Recomenda-se, portanto, não retirar o bebê em um tempo muito curto, após ser colocado na posição canguru, devendo permanecer pelo menos durante uma hora, sendo considerado o tempo para se organizar e depois entrar em sono profundo, o que é muito importante para o desenvolvimento cerebral e sua recuperação ${ }^{(13)}$.

Com esse fato, à proporção que os pais se sentem livres para permanecer o tempo que lhes é possível com seu RN assistido em unidades neonatais, eles ficam mais confiantes para cuidar, melhorando a qualidade do relacionamento mãe-filho-família. Neste momento, torna-se fundamental a presença dos profissionais técnicos de enfermagem no auxílio dos pais, não só nos cuidados, como também no apoio emocional ${ }^{(21)}$.

Contudo, assim como evidenciado nos depoimentos das entrevistadas, quanto ao excesso de manuseios do RN, os profissionais também devem estimular o manuseio só quando necessário. Esta é uma questão a ser observada, uma vez que provoca alterações fisiológicas, estresse e desconforto; pois os bebês prematuros nesta fase da vida são muito frágeis e passíveis de desenvolvimento de diversas complicações ${ }^{(19)}$ Portanto, a redução da manipulação e uma das medidas essenciais para se prevenir e manejar a dor no neonato.

A organização do bebê, quanto ao uso dos "acolchoados" para a formação de ninhos ("rolinhos"), foi abordada por 17 das participantes como sendo uma importante ferramenta também. O posicionamento com os acolchoados foi entendido como essencial porque o prematuro não pode manter uma posição confortável por si próprio, logo um mau posicionamento, com o tempo, pode afetar seu desenvolvimento motor.

Quando o neonato é posicionado na UTI neonatal em diversas posições, experimenta uma variedade de pressões e forças nos músculos e articulações, que vão influenciar positivamente no desenvolvimento destas e dos receptores mecânicos, preparando, assim, para movimentos mais coordenados $^{(18)}$.

Foram evidenciadas, no presente estudo, as dificuldades de aplicabilidade do método na unidade neonatal, visto que 12 (60\%) das entrevistadas ressaltaram que encontram dificuldades na aplicabilidade devido à capacitação insuficiente e ao espaço físico inadequado para sua implementação, como observado a seguir:

“[...] Deveria ter outra capacitação e mais espaço físico. Acredito que deve se ter um maior treinamento da equipe e um melhor espaço físico para assim ter melhor adesão do método" (T8).

"[...] tenho dificuldade de aplicar o método pela falta de material e espaço, que é diminuido, acho que deveria ter mais cursos, mais informações, pois às vezes se perde com a rotina e com o tempo" (T10).

"[...] acho que deveria ver mais treinamento, pois sei que nem todo mundo faz. Enquanto faço de um jeito outras pessoas fazem diferente e isso não fortalece o método. (T17)

Os pontos mais ressaltados nos discursos foram a falta de capacitação e a necessidade de melhoras da estrutura física, o que, segundo as depoentes, contribui para aumentar as dificuldades de uma prática comprometida com a singularidade do $\mathrm{RN}$.

Uma das principais dificuldades para a implantação do MC é a adesão da equipe de saúde que atua com RN prematuros. Para tanto, uma série de medidas estão sendo implantadas pelo MS, que desde 1999 implantou em todo o Brasil a "Norma de Atenção Humanizada ao RecémNascido prematuro de baixo peso-Método canguru". Desde então, o MS vem desdobrando esforços na tentativa de difundir o MC e capacitar os profissionais envolvidos na assistência ao RN. Por outro lado, a adesão dos profissionais ainda não é efetiva, mas pode ser facilitada se as evidências das vantagens do MC forem consolidadas ${ }^{(10)}$.

Com a implementação de estratégias gerenciais para efetivação do $\mathrm{MC}$, qualquer unidade de saúde, desde as menos equipadas, com pouca tecnologia disponível, até as mais complexas, podem implementar o MC em suas rotinas com anuência de poucos recursos, não havendo necessidade de se esperar por mudanças estruturais, embora elas devam ser sempre buscadas visando a melhoria do ambiente $\operatorname{assistencial}^{(7,10,20)}$.

O presente estudo apresentou como fator limitante o fato de se restringir a uma realidade investigada entre um número pequeno de profissionais de uma unidade específica de neonatologia do estado do Pará, impossibilitando assim que se possa estabelecer maiores generalizações a um grupo maior de indivíduos, necessitando, assim, de futuras pesquisas para um melhor aprofundamento sobre a temática.

\section{CONSIDERAÇÕES FINAIS}

O estudo evidenciou que os técnicos de enfermagem entrevistados possuem conhecimentos sobre o $\mathrm{MC}$, no entanto, apontam dificuldades quanto à identificação das etapas e a sua aplicabilidade em seus cotidianos, sendo estas representadas aqui devido às faltas de maiores capacitações profissionais na área, a escassez de recursos e a estrutura física deficitária no cotidiano da prática neonatal. 
Apesar das dificuldades encontradas, observa-se a valorização da importância do $\mathrm{MC}$ na assistência dentro da UTI neonatal no que tange à recuperação, crescimento e desenvolvimento do $\mathrm{RN}$, mostrando uma elevação da satisfação do cuidado oferecido. Dessa forma, acredita-se que não basta somente o técnico de enfermagem conhecer sobre o método, são necessárias maiores articulações que os possibilite repassar valores e conhecimento do método para sua clientela assistida.

\section{REFERÊNCIAS}

1. Spehar MC, Seidl EMF. Percepções maternas no Método Canguru: contato pele a pele, amamentação e autoeficácia. Psicol Estud. 2013;18(4):647-56.

2. Almeida H, Venancio SI, Sanches MTC, Onuki D. Impacto do método canguru nas taxas de aleitamento materno exclusivo em recém-nascidos de baixo peso. J Pediatr. 2010;56(3):250-3.

3. Arivabene JC, Tyrrell MAR. Kangaroo mother method: mothers' experiences and contributions to nursing. Rev Latino-Am Enfer. 2010;18(2):262-8.

4. Gontijo TL, Xavier CC, Freitas MIF. Avaliação da implantação do Método Canguru por gestores, profissionais e mães de recém-nascidos. Cad Saúde Pública. 2012;28(5):935-44.

5. Borck M, Santos EKA. Método canguru: práticas investigativas e de cuidado de enfermagem no modelo de adaptação de Roy. Esc Anna Nery Rev Enferm. 2012;16(2):263-9.

6. Rodrigues MAG, Cano MAT. Estudo do ganho de peso e duração da internação do recém-nascido pré-termo de baixo peso com a utilização do método canguru. Rev Eletrônica Enferm. 2006;8(2):185-91.

7. Veras RM, Vieira JMF, Morais FRR. A maternidade prematura: o suporte emocional através da fé e religiosidade. Psicol Estud. 2010;15(2):325-32.

8. Hennig MAS, Gomes MASM, Morsch DS. Atenção humanizada ao recém-nascido de baixopeso. Método Canguru e cuidado centrado na família: correspondências e especificidades. Physis. 2010;20(3):835-52.

9. Santos L, Morais R, Freitas MJ, Santana R, Oliveira V, Nery F. Maternal perception of the skin to skin contact with premature infants through the kangaroo position. Rev Pesqui. Cuid Fundam. 2013;5(1):3504-14.

10. Ministério da Saúde (BR). Atenção á saúde do recémnascido: guia para os profissionais de saúde. Brasília: Ministério da Saúde; 2011.
11. Cabral IE, Groleau D. Breastfeeding practices after Kangaroo Mother Method in Rio de Janeiro: the necessity for he alth education and nursing intervention at home. Esc Anna Nery Rev Enferm. 2009;13(4):76371.

12. Ministério da Saúde (BR), Secretaria de Políticas de Saúde, Área da Saúde da Criança. Atenção humanizada ao recém-nascido de baixo peso: método mãe-canguru. Brasília: Ministério da Saúde; 2009.

13. Neves PN, Ravelli APX, Lemos JRD. Atenção humanizada ao recém-nascido de baixo-peso (método Mãe Canguru): percepções de puérperas. Rev Gaúcha Enferm. 2010;31(1):48-54.

14. Creswell JW. Qualitative inquiry and research design: Choosing among five approaces. $3^{\text {th }}$ ed. California: Sage; 2012.

15. Fontanella BJB, Magdaleno Júnior R. Saturação teórica em pesquisas qualitativas: contribuições psicanalíticas. Psicol Estud. 2012;17(1):63-71.

16. Teixeira E. As três metodologias: acadêmica, da ciência da pesquisa. $5^{\text {a }}$ ed. Belém: Vozes; 2009.

17. Veras MR, Traverso YMA. A maternidade na política de humanização dos cuidados ao bebê prematuro e/ ou de baixo peso: Programa Canguru. Rev Estud Fem. 2010;18(1):61-80.

18. Araújo CL, Rios CTF, Santos MH, Gonçalves APF. Método Mãe Canguru: uma investigação da prática domiciliar. Ciênc Saúde Coletiva. 2010;15(1):301-7.

19. Borck M, Santos EKA. Terceira etapa método Canguru: convergência de práticas investigativas e cuidado com famílias em atendimento ambulatorial. Rev Gaúcha Enferm. 2010;31(4):761-8.

20. Gontijo TL, Meireles AL, Malta DC, Proietti FA, Xavier CC. Evaluation of implementation of humanized care to low weight newborns - the Kangaroo Method. J Pediatr. 2010;86(3):33-9.

21. Sousa BB, Santos MH, Sousa FGM, Gonçalves APF, Paiva SS. Avaliação da dor como instrumento para cuidar de recém-nascidos pré-termo. Texto \& Contexto Enferm. 2006;15(Esp):88-96.

\section{Endereço para correspondência:}

Rosangela Aviz Silva

Universidade do Estado do Pará (UEPA)

Rua do Uma, 156

Bairro: Telégrafo

CEP: 66050-540 - Belém - PA - Brasil

E-mail: roaviz@yahoo.com.br 